\title{
Expression of base excision repair key factors and miR17 in familial and sporadic breast cancer
}

\author{
S De Summa ${ }^{1}$, R Pinto ${ }^{1}$, B Pilato ${ }^{1}$, D Sambiasi ${ }^{1}$, L Porcelli ${ }^{2}$, G Guida ${ }^{3}$, E Mattioli ${ }^{4}$, A Paradiso ${ }^{5}$, G Merla ${ }^{6}$, L Micale ${ }^{6}$, P De Nittis ${ }^{6}$ and \\ S Tommasi, ${ }^{*}$
}

Understanding of BRCA1/2 interaction with the base excision repair (BER) pathway could improve therapy based on 'synthetic lethality', whose effectiveness is based on homologous recombination deficiency in cells lacking functional BRCA genes. However, poly (ADP-ribose) polymerase (PARP) inhibitors failed in some patients and for this reason we explored BER key enzyme expression. In this study, the expression of BER enzymes (redox factor 1/apurinic-apyrimidinic endonuclease 1 (REF1/ APEX1), NTH endonuclease III-like 1 (NTHL1), 8-oxoguanine DNA glycosylase (OGG1), PARP1) and of the scaffold protein XRCC1 (X-ray repair complementing defective repair in Chinese hamster cells 1) were investigated in familial (BRCA-related and not) and sporadic breast cancer cases. Furthermore, miR17 expression was measured because of its role in the epigenetic regulation of BRCA1. Gene expression was evaluated in BRCA1-mutated cell lines, SUM149PT and SUM1315MO2, and in a BRCA1-proficient triple-negative MDA-MB-231 cell line. A cohort of 27 familial and 16 sporadic breast cancer patients was then examined to confirm results obtained from the cell line model. APEX1/REF1 was found to be upregulated in familial BRCA-wild-type and sporadic cases, indicating this enzyme as a potential therapeutic target. Furthermore, XRCC1 was overexpressed in BRCAX patients; consequently, we suggest to test the effectiveness of inhibitors targeting two different BER components in preclinical studies. XRCC1, which is also involved in the non-homologous end-joining pathway, was found to be downregulated in BRCA2related patients concurrently with no change in PARP1 expression. Interestingly, no difference in PARP1 and miR17 expression was found in BRCA-related and sporadic breast cancer cases. PARP1 and miR17 could therefore be further investigated as molecular biomarkers of 'BRCAness' phenotype, indicating patients which could really benefit from PARP inhibitor therapies. Cell Death and Disease (2014) 5, e1076; doi:10.1038/cddis.2014.30; published online 20 February 2014

Subject Category: Cancer

Cancer cells contain multiple mutations and chromosomal aberrations, ${ }^{1,2}$ which can confer them growth advantage, principally owing to their accumulation. ${ }^{3}$ In fact, carcinogenesis is strictly associated with DNA lesion accumulation and genomic instability. Therefore, genome surveillance machinery has great importance in the repair of DNA damage. This damage is caused by a combination of defective genetic material and environmental insults such as ionizing or ultraviolet radiation, chemicals, or reactive oxygen species, which are also produced during normal metabolic processes.

Breast cancer develops from a heterozygous population of diseases of the breast, which are intimately related to DNA damage repair defects or defects in cell-cycle checkpoints. Aberrant expression of DNA damage response genes is common in nearly all breast cancer phenotypes, ${ }^{3}$ while only
$20-30 \%$ of breast cancer cases are thought to be caused by germline mutations in BRCA1/2 susceptibility genes. ${ }^{4-6}$

BRCA1/2 proteins take part in different protein complexes acting in DNA damage response, such as homologous recombination (HR), non-homologous end-joining (NHEJ), and nucleotide excision repair (NER), but the significance of such interactions has not yet been completely clarified. ${ }^{7,8}$ Principally, BRCA1 has an early role, together with BRCA2 and RAD51, in the promotion and regulation of $\mathrm{HR}$, and is part of BASC (BRCA1-associated genome surveillance complex), which influences the choice of repair pathway depending upon the type of DNA lesion. ${ }^{9}$ Thus, cells carrying BRCA1 mutations are deficient in the transcription-coupled repair of oxidative damage. ${ }^{10}$ Moreover, BRCA1 could also be involved in other DNA repair pathways, for example, promoting NHEJ

\footnotetext{
${ }^{1}$ Molecular Genetics Laboratory, National Cancer Research Centre, Istituto Tumori 'Giovanni Paolo II', Bari, Italy; ${ }^{2}$ Preclinical and Clinical Pharmacology Laboratory, National Cancer Research Centre, Istituto Tumori 'Giovanni Paolo II', Bari, Italy; ${ }^{3}$ Department of Medical Biochemistry, Medical Biology and Medical Physics, School of Medicine, University of Bari, Bari, Italy; ${ }^{4}$ Anatomopathology Unit, National Cancer Research Centre, Istituto Tumori 'Giovanni Paolo II', Bari, Italy; ${ }^{5}$ Experimental Medical Oncology Unit, National Cancer Research Centre, Istituto Tumori 'Giovanni Paolo II', Bari, Italy and ${ }^{6}$ Medical Genetics Unit, IRCCS Casa Sollievo delle Sofferenza, San Giovanni Rotondo, Italy

*Corresponding author: S Tommasi, Molecular Genetics Laboratory, National Cancer Research Centre, Istituto Tumori 'Giovanni Paolo Il', v.le Orazio Flacco 65, Bari 70124, Italy. Tel: +39 0805555 283; Fax: +39 0805555 388; E-mail: s.tommasi@oncologico.bari.it

Keywords: base excision repair; APEX1/REF1; PARP1; miR17; XRCC1; familial breast cancer

Abbreviations: BER, base excision repair; HR, homologous recombination; NHEJ, non-homologous end-joining; NER, nucleotide excision repair; AP, apurinicapyrimidinic; TNBC, triple-negative breast cancer; REF1/APEX1, redox factor 1/apurinic-apyrimidinic endonuclease 1; NTHL1, NTH endonuclease III-like 1; OGG1, 8-oxoguanine DNA glycosylase; XRCC1, X-ray repair complementing defective repair in Chinese hamster cells 1; PARP-1, poly (ADP-ribose) polymerase 1; IDC, invasive ductal carcinoma; ILC, invasive lobular carcinoma; ER, estrogen receptor; PgR, progesterone receptor; HER2, human epidermal growth factor receptor 2 Received 9.7.13; revised 24.12.13; accepted 2.1.14; Edited by G Ciliberto
} 
in DNA double-strand break repair ${ }^{11}$ or NER, ${ }^{12}$ or influencing base excision repair (BER). ${ }^{13}$

In brief, the BER pathway consists of enzymatic steps involving the following: 8-oxoguanine DNA glycosylase (OGG1) and NTH endonuclease III-like 1 (NTHL1), DNA glycosylases that recognize specific subsets of damaged bases and that prefer substrates such as 8-oxoguanine and thymine-glycol lesions; REF1/APEX1 (redox factor 1/apurinicapyrimidinic endonuclease 1), a multifunctional enzyme with apurinic-apyrimidinic (AP) endonuclease activity and $3^{\prime}-5^{\prime}$ exonuclease, $3^{\prime}$-diesterase, and $3^{\prime}$-phosphatase activities; a DNA polymerase and a DNA ligase. Furthermore, XRCC1 (Xray repair complementing defective repair in Chinese hamster cells 1), a scaffold protein, is able to associate with several other proteins (polynucleotide kinase, DNA polymerase $\beta$, and DNA ligase III) to form a complex that repairs the single-strand DNA breaks generated during the BER process. Poly-ADP ribose polymerases (PARPs) are a family of enzymes that catalyze polymerization and formation of highly negatively charged poly-ADP ribose chains. A key role for PARP1 and PARP2 is maintaining genomic integrity, in particular repair of single-strand DNA lesions through the BER pathway. Recently, the efficiency of the BER pathway has been studied in relation to mutations or the altered expression of $B R C A 1 / 2$ genes. In cell lines carrying mutations in BRCA2 (Capan-1 cell line, human pancreatic adenocarcinoma cell line), ${ }^{14}$ a reduced rate of DNA ligation during both single-nucleotide insertion and the PCNA-dependent pathway of BER ${ }^{15}$ was found; in MCF-7 and T47D cell lines (human mammary adenocarcinoma cell lines), BRCA1 overexpression caused a twofold increase in the mRNA levels of OGG1, NTHL1, REF1/APEX1, and XRCC1. ${ }^{13}$

Taking evidence obtained in previous studies as a starting point, in this study gene expression of BER factors that seemed to be transcriptionally regulated by BRCA1 was measured together with the expression of PARP1, considering that the use of PARP inhibitors is still controversial.

However, as BRCA1 loss of function is correlated not only to mutations but also to epigenetic changes, such as promoter methylation ${ }^{16}$ and microRNA regulation, the expression of miR17, a validated regulator of BRCA1, ${ }^{17}$ was measured to confirm this association with respect to BER key enzyme expression. miR17 expression has been explored not only because of its role in regulating $B R C A 1$ but also because computational analysis indicated it as a regulator of APEX1/REF1.

This is the first study that aims to analyze the association between BER gene transcription and BRCA mutational status, to better understand the ongoing preclinical studies regarding synthetic lethality through PARP inhibition and other BER factors, such as APEX1/REF1.

\section{Results}

BER genes and miR17 expression in cell lines. In this study, APEX1/REF1, NTHL1, OGG1, XRCC1, PARP1, and miR17 expression was preliminarily explored in two BRCA1mutated cell lines, SUM149PT and SUM1315MO2, and in the BRCA1-proficient triple-negative (estrogen receptor
(ER)-, progesterone receptor (PgR)-, human epidermal growth factor receptor 2 (HER2)/Neu-negative) MDA-MB231 cell lines. APEX1/REF1, OGG1, and XRCC1 were found to be downregulated in all cell lines (Figure 1). Notably, NTHL1 was upregulated in SUM149PT and MDA-MB-231, classified as basal- and mesenchymal-like, respectively, by Lehmann et al., ${ }^{18}$ but not in ER-positive SUM1315MO2, suggesting the existence of other regulatory mechanisms for this enzyme. miR17 was found to be upregulated in mesenchymal-like MDA-MB-231 and in BRCA1-mutated SUM1315O2 cell lines. Interestingly, overexpression of PARP1 was also found in MDA-MB-231 cells (Figure 1). The median fold change of BER genes was compared in BRCA1- and BRCA2-mutated patients. Although no statistical analysis could be performed because of the small dimension of the two subsets, interestingly we observed a greater downregulation of APEX1/REF1, NTHL1, and $\mathrm{XRCC} 1$, and an upregulation of PARP1 and miR17 in BRCA1-mutated patients, giving us further confirmation of what was found in the cell lines (Figure 2).

Upregulation of APEX1/REF1 in BRCAX and sporadic patients. In our cohort of patients, the APEX1/REF1 median fold change was significantly higher in sporadic patients than in familial cases $(P=0.0176)$ (Figure 3a). Moreover, considering the mutational status of $B R C A 1 / 2$ genes, APEX1/ REF1 was overexpressed in BRCAX patients $(P=0.0057)$ and in sporadic samples versus mutated ones $(P=0.0108)$ (Figures $3 \mathrm{~b}$ and $\mathrm{c}$ ). These findings were also confirmed when analyzing frequencies of overexpressing patients, considering the median fold change as the cutoff level. We observed that $68.8 \%(P=0.004)$ and $76.9 \%(P=0.002)$ of sporadic and BRCAX patients, respectively, showed upregulation of APEX1/REF1 compared with mutated cases (Figures $3 d$ and f).

PARP1 and miR17 expression. In our cohort, we found PARP1 fold change in the opposite direction in BRCA1-and BRCA2-mutated patients (Figure 2). Notably, we found a significant upregulation of PARP1 in mutated patients versus $\mathrm{BRCAX}(P=0.0015)$ and in sporadic patients versus BRCAX $(P=0.0190)$ (Figures $4 a$ and b). Interestingly, the same behavior was observed in miR17 expression also in terms of the frequencies of overexpressing patients (data not shown).

miR17 computational analysis: APEX1/REF1 predicted target. To identify miR17 targets, a computational analysis was performed with the miRWalk database. As expected, miR17 was validated to bind BRCA1-3'-UTR $(P=0.0053)$. Interestingly, APEX1/REF1 was identified as a predicted target of miR17 with a good level of significance $(P=0.0172)$ (Table 1). When analyzing the miRNA in our panel of cell lines, overexpression of miR17 together with a downregulation of APEX1/REF1 was found in MDA-MB-231 and SUM1315MO2 cell lines. However, in the BRCA1-mutated SUM149PT, miR17 resulted slightly downregulated in the presence of heavy APEX1/REF1 downregulation, suggesting that in these cells a different genetic or epigenetic regulation can occur (Figure 1). 


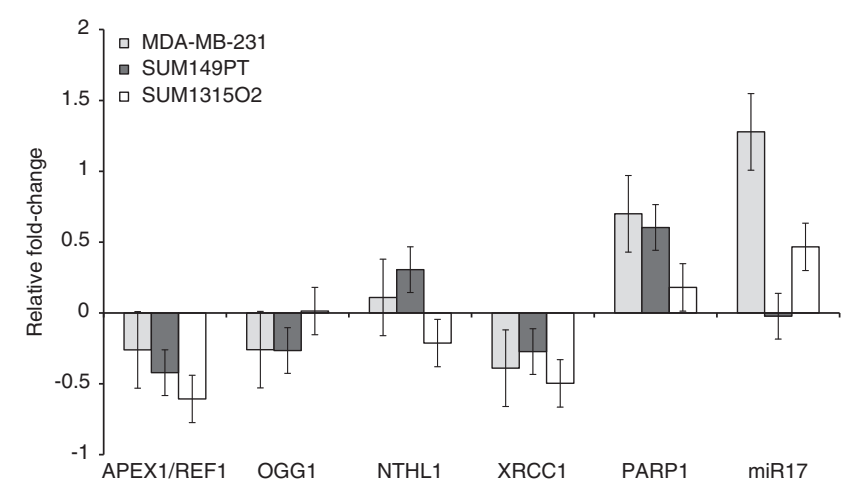

Figure 1 Relative expression \pm S.D. (evaluated as $\log _{2}$ median fold change) of $B E R$ genes and miR17 in BRCA1-proficient triple-negative MDA-MB-231- and BRCA1-mutated cell lines, SUM149PT and SUM1315MO2 (triple-negative and ERpositive, respectively). All experiments were replicated three times

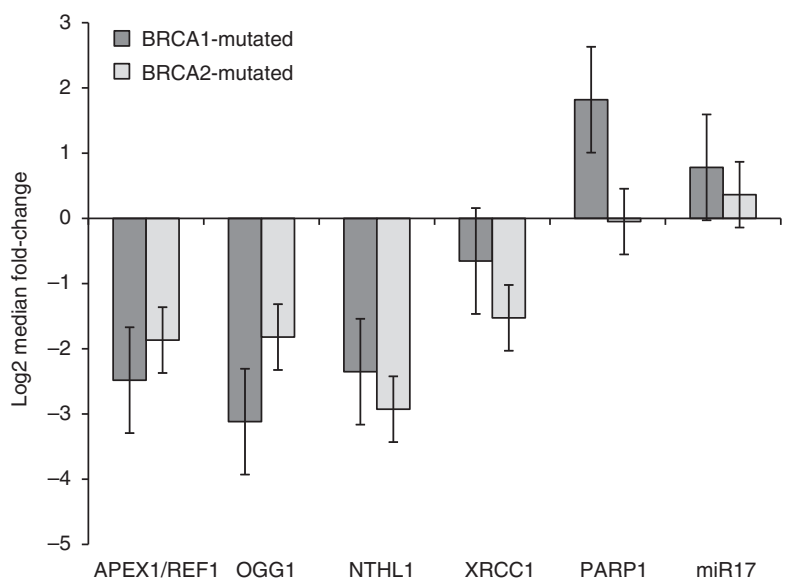

Figure 2 Relative expression \pm S.D. (evaluated as $\log _{2}$ median fold change) of $B E R$ genes and miR17 in 10 BRCA1- and 4 BRCA2-related breast cancer samples. Data were normalized as described in Materials and Methods. Briefly, equimolar pools of healthy tissues of BRCA1- and BRCA2-related breast cancer were used to calibrate gene expression after normalization with endogenous control. All experiments were replicated three times

In our cohort, an inverse correlation between APEX1/REF1 and miR17 was found both in BRCAX and sporadic patients, even if this result was not statistically significant (data not shown).

Functional analysis to test miR17 regulation of APEX1/ REF1. To test whether miR-17 directly target the $3^{\prime}$-UTR of APEX1, HEK293 cells were co-transfected with APEX1 reporter construct along with a synthetic mimic of miR-17. As shown in Figure 5, the overexpression of miR-17 did not have any significant effect on the luciferase activity of the vector containing the $3^{\prime}-$ UTR of APEX1 when compared with the control. Consistently, deletion of the binding site and the use of specific miR-17 inhibitor did not have any consequence on the APEX1 level (Figure 5).

XRCC1, NTHL1, and OGG1 transcript abundances. We observed a significant upregulation of glycosylases, OGG1 and NTHL1 ( $P=0.0097$ and 0.0165 , respectively), in wildtype patients versus mutated cases. These data were confirmed in terms of the percentage of overexpressing patients: $92.3 \%$ of BRCAX cases overexpressed both genes $(P=0.0001)$ (data not shown).

Furthermore, the XRCC1 median fold change was significantly higher in BRCAX tissues versus mutated patients $(P=0.0308)$ and $76.9 \%$ of BRCAX cases overexpressed XRCC1 when compared with mutated ones $(P=0.035)$ (Figure 6).

\section{Discussion}

In this study, we focused on BER key enzyme transcriptional levels to understand if their expression could be influenced by BRCA mutational status or if they behave differently in familial or sporadic breast cancer cases. Saha et al. ${ }^{13}$ reported that BRCA1 was able to stimulate transcriptionally several early steps in the BER pathway, in particular those in which APEX1/ REF1, OGG1, NTHL1, and XRCC1 are involved. Clarification on this pathway is important because of its involvement in synthetic lethality. In fact, it has been shown that HR-deficient tumors, including those with defects in BRCA1/2 genes, are very sensitive to a blockade of the BER pathway via inhibition of the PARP enzyme. ${ }^{18}$ Thus, a better understanding of the BER pathway has implications in the potentiation of the therapeutic effects of DNA-damaging agents.

Preliminarily, we measured APEX1/REF1, OGG1, NTHL1, and PARP1 transcript levels in two BRCA1-mutated cell lines (SUM149PT and SUM1315MO2) and in the BRCA1-proficient triple-negative MDA-MB-231 cell line (ER-, PgR-, HER2/Neunegative). The downregulation of APEX1/REF1, OGG1, and XRCC1 confirmed the results of Saha et al. ${ }^{13}$ In particular, the overexpression of PARP1 and miR17, which is a validated regulator of $B R C A 1$, in the mesenchymal-like MDA-MB-231 cell line, together with results reported on the sensitivity of MDA-MB-231 to the PARP inhibitor ABT-888 (veliparib), ${ }^{19}$ prompted us to enroll a cohort of sporadic breast cancers independently of immunophenotype (basal, mesenchymal- or luminal-like) and hormone receptor, and HER2 status. A series of familial breast cancers, BRCA1/2-related and BRCAX tumors, was also analyzed to confirm the expression data measured in the cell line model (Figure 1).

Interesting results were found regarding the expression of APEX1/REF1. In particular, the median fold change was significantly higher in BRCAX and sporadic cases with respect to mutated ones, and a significant percentage of them $(76.9 \%$ and $68.8 \%$, respectively) overexpressed APEX1/REF1 (Figure 3). Altered expression of APEX1/REF1 was previously observed in gliomas, ${ }^{20}$ rhabdomyosarcomas, ${ }^{21}$ and non-small-cell lung cancer, ${ }^{22}$ and its overexpression was correlated with poor outcome, ${ }^{23}$ poor complete response rate to radiotherapy, ${ }^{24}$ poorer survival, ${ }^{25}$ and higher angiogenesis. ${ }^{26}$ The active site for AP endonuclease, $3^{\prime}-5^{\prime}$ endonuclease, 3'-phosphatase, and $3^{\prime}$-phopshodiesterase activities is located at the C-terminal domain of the protein, making the identification of small-molecule inhibitors for this protein easier. Luo et al. ${ }^{27}$ inhibited APEX1/REF1 endonuclease activity through lucanthone in a breast cancer MDAMB-231 cell line, increasing the cytotoxicity of temozolomide 

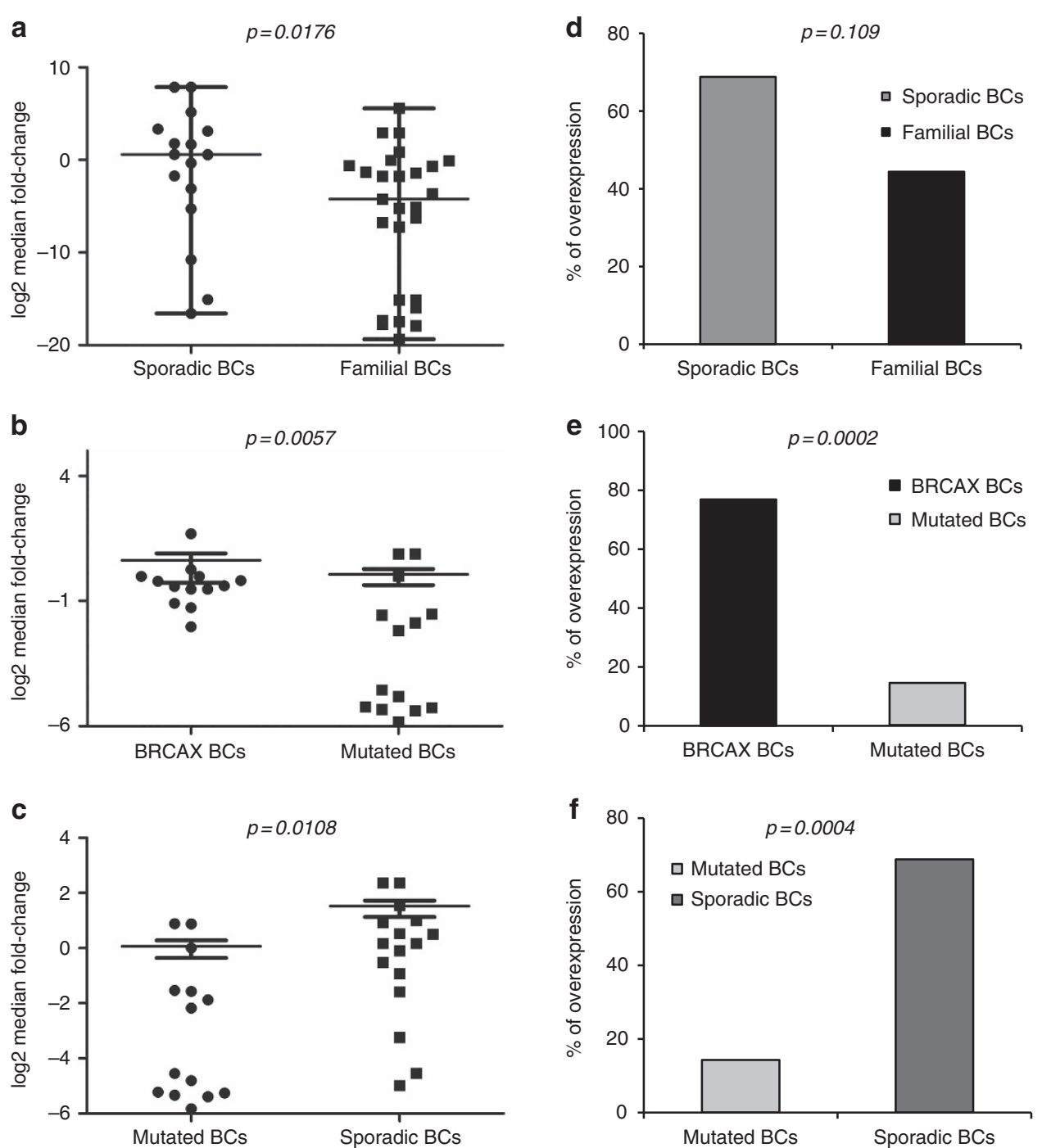

Figure 3 Relative expression (evaluated as $\log _{2}$ median fold change) calculated through Mann-Whitney U-test and percentage of overexpression (considering median of $\mathrm{e}^{-\Delta \Delta \mathrm{Ct}}$ values as cutoff level) of APEX1/REF1 in (a-d) sporadic versus familial; (b-e) BRCAX versus mutated; (c-f) mutated versus sporadic patients. The level of significance has been reported for each comparison. Data were considered significant when $P<0.05$. All experiments were replicated three times. BC, breast cancer

(TMZ), while in other studies overexpression of APEX1/REF1 in glioma cell lines was correlated with high resistance to radiotherapy. ${ }^{23,24}$ The DNA lesions caused by TMZ, which are mostly the methyl adducts N7-methylguanine and N3methyladenosine, become highly cytotoxic when BER is impaired. ${ }^{28}$ Considering the present data and the unique role of APEX1/REF1 in repairing AP sites, ${ }^{29,30}$ this gene could be considered a useful therapeutic target (e.g., through lucanthone or methoxyamine) in the absence of BRCA mutations (BRCAX cases) and in sporadic patients to enhance the therapeutic benefit of DNA-damaging agents, such as TMZ or radiotherapy. Computational analysis indicated APEX1/REF1 as a predicted target of miR17. The present data showed a significant overexpression of miR17 in the BRCA-related subset, which had lower expression of APEX1/REF1. For this reason, a luciferase assay was performed to confirm bioinformatic prediction and eventually to target APE1/REF1 with miRNA mimic strategy. However, our experimental data showed that miR-17 does not regulate APEX1 expression, at least by the direct binding to the $3^{\prime}$-UTR region of APEX1 (Figure 5).

As expected, PARP1 was found to be upregulated in mutated cases, but interestingly it was more overexpressed in BRCA1-related patients than in BRCA2-related ones (Figure 2). OGG1 gene is more downregulated than APE1/ REF1 and, more interestingly, its expression level is lower in BRCA1 - than in BRCA2-related breast cancers (Figure 2). Alli et $a .^{31}$ reported that knockdown of OGG1 confers sensitivity to PARP inhibitors, indicating this gene as possible prognostic factor and therapeutic target. These data have to be further investigated to understand if patients have to be stratified, taking into account in which gene (BRCA1 or BRCA2) they carry a deleterious mutation, which would then allow us to assess the reason for the different responsiveness of BRCA1 and BRCA2 mutation carriers to PARP inhibitors. ${ }^{32}$ Many clinical trials are ongoing to evaluate PARP inhibitor 

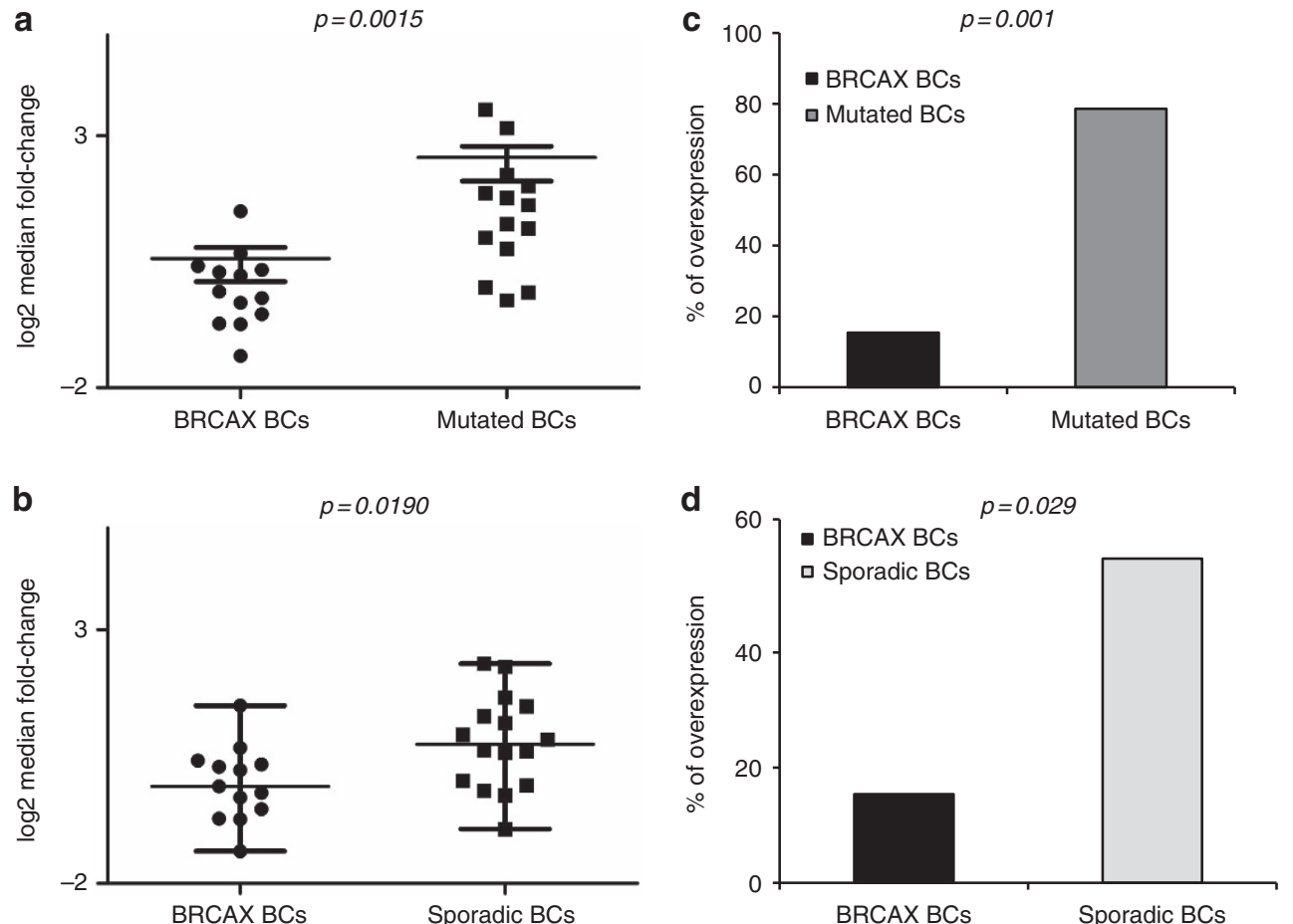

Figure 4 Relative expression (evaluated as $\log _{2}$ median fold change) calculated through Mann-Whitney U-test and percentage of overexpression (considering median of $e^{-\Delta \Delta C t}$ values as cutoff level) of PARP1 in (a-c) BRCAX versus mutated; $(\mathbf{b}-\mathbf{d})$ BRCAX versus sporadic patients. The level of significance has been reported for each comparison. Data were considered significant when $P<0.05$. All experiments were replicated three times

Table 1 Computational analysis results performed with miRwalk

\begin{tabular}{lcccccrrr}
\hline & Gene name & RefSeqID & MicroRNA & Start & Sequence & End & Region & P-value \\
\hline (A) & BRCA1 & NM_007295 & hsa-miR-17 & 6604 & CAAAGUGCU & 6596 & 3'-UTR & 0.0053 \\
\hline (B) & APEX1 & NM_001641 & hsa-miR-17 & 1310 & CAAAGUG & 1304 & $3^{\prime}$-UTR & 0.0172
\end{tabular}

(A) BRCA1 has been validated as has-miR-17 target and (B) new target for has-miR-17 has been evidenced

effectiveness in breast cancer cases, which display the socalled 'BRCAness' phenotype - breast cancers that are not BRCA-related but that display the same phenotype, such as HR deficiency. However, patients recruited into the trials are generally triple-negative breast cancer (TNBC) for hormone receptors (ER, PgR) and HER2, and, to date, results have not been encouraging. ${ }^{33}$ Goncalves et al. ${ }^{34}$ showed PARP1 mRNA overexpression not only in TNBCs but also in other types of breast tumor, mainly basal-like, and also in luminal types. In our cohort, BRCA1/2-related TNBCs overexpressed PARP1. It is important to underline, however, that the sporadic patients analyzed in our study were not TNBCs, except for one case that did not overexpress PARP1. BRCA1 dysfunction has been reported in basal-like sporadic cancers, suggesting sensitiveness to PARP1 inhibition. ${ }^{35}$ PARP1 inhibition effectiveness in other subtypes of breast cancer is ongoing, but a better stratification of patients is mandatory. Notably, miR17 expression data showed similar results to PARP1, also in terms of the frequency of overexpressing patients, and in particular as regards wild-type versus BRCA-related subjects. The significant overexpression of miR17 in sporadic patients seems to suggest the downregulation of BRCA1, mimicking a 'BRCAness' phenotype. Furthermore, no statistically significant difference in PARP1 and miR17 was observed between the sporadic and mutated cases, suggesting a need to validate miR17 and PARP1 as molecular biomarkers of BRCAness phenotype in a larger cohort. Upregulation of PARP1 and miR17 in sporadic versus BRCAX patients was also observed. Such a preliminary observation seemed to give further confirmation that their overexpression, similar to what was observed in BRCA-mutated cases, could mimic BRCAness phenotype (Figure 4b).

XRCC1 is a scaffold protein involved in the coordination of DNA repair by interacting with several components of the BER and single-strand break repair pathways, such as DNA glycosylases, APEX1/REF1, PARP1, PNK, and ligllI. ${ }^{36}$ In all 


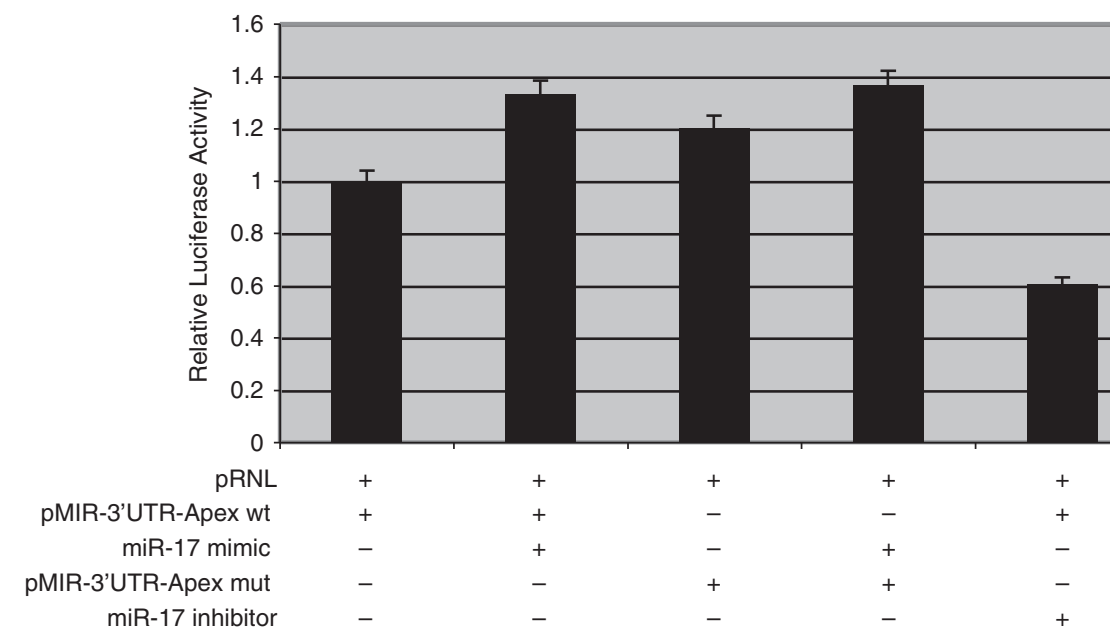

Figure 5 HEK293 cells were co-transfected with reporter constructs carrying the $3^{\prime}$-UTR of APEX 1 or $3^{\prime}$-UTR of APEX 1 containing mutated miR-17 complementary site or a synthetic mimic of miR-17 or a miR-17 inhibitor. Luciferase activities were measured and normalized to the level of control Renilla luciferase
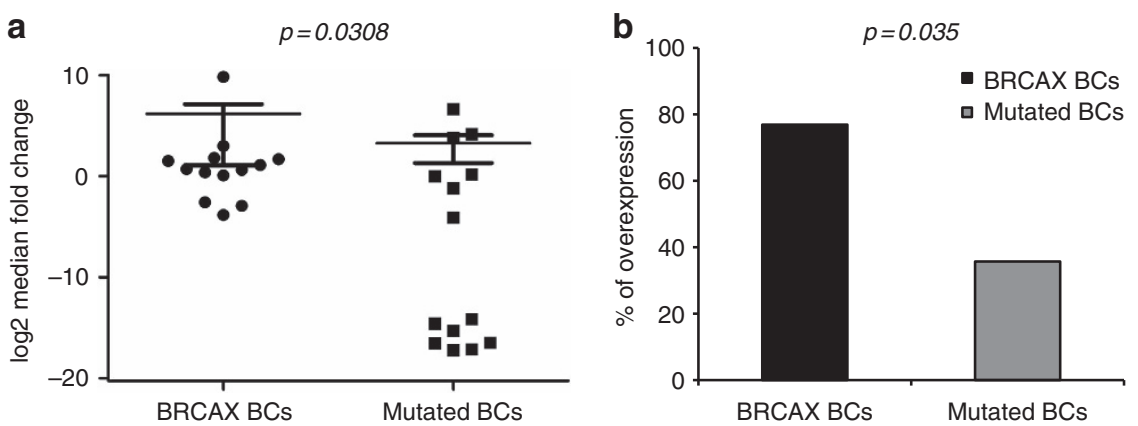

Figure 6 Relative expression (evaluated as $\log _{2}$ median fold change) calculated through Mann-Whitney U-test and percentage of overexpression (considering median of $\mathrm{e}^{-\Delta \Delta C \mathrm{Ct}}$ values as cutoff level) of XRCC1 in BRCAX versus mutated patients. The level of significance has been reported for each comparison. Data were considered significant when $P<0.05$. All experiments were replicated three times

cell lines of our model, XRCC1 was found to be downregulated and, in the present cohort of patients, BRCAX cases overexpressed, as compared with BRCA-related patients. Furthermore, $92.3 \%$ of BRCAX displayed OGG1 and NTHL1 overexpression. This suggests an activation of the BER pathway as it is well known that XRCC1 stimulates the activity of glycosylases. ${ }^{37}$ XRCC1 transcript level was negatively correlated with cisplatin cytotoxicity in non-small-cell lung cancer cell lines, ${ }^{38}$ and downregulation of XRCC1 through shRNA strategy in HepG2 cells inhibited double-strand break repair, enhancing cisplatin cytotoxicity. Thus, XRCC1 could eventually be considered as a molecular target to enhance the effects of DNA-damaging agents in BRCAX patients (Figure 6).

In invasive breast cancer, loss of XRCC1 has recently been indicated as an independent predictor of poor clinical outcome. $^{39}$ XRCC1 is also a key component of the NHEJ pathway ${ }^{40,41}$ and the complex interaction existing between NHEJ and HR is emerging. ${ }^{42}$ Sultana et al. ${ }^{39}$ evaluated sensitivity to inhibitors of ATM (a sensor of DNA damage involved in HR) and DNA-PKcs (a component of NHEJ) in XRCC1-deficient cell lines. Peasland et al. ${ }^{43}$ showed cytotoxicity of an ATR inhibitor in cells lacking XRCC1. These preclinical studies seemed to indicate that XRCC1-deficient cells could be targeted by blocking double-strand break repair pathways. Figure 2 reported the downregulation of XRCC1 and the unchanged expression of PARP1 in BRCA2-related patients. These data evidenced a basal activity of BER in contrast to what was observed in BRCA1-mutated cases. Furthermore, taking into account these findings and the previously described preclinical studies, it could be possible to treat differently BRCA1- and BRCA2-mutated patients targeting PARP1 and NHEJ pathway, respectively.

In conclusion, differential expression of BER key enzymes could be useful to better stratify breast cancer to improve target therapies targeting BER components other than PARP1. In particular, the results suggested considering APEX1/REF1 as a molecular target in BRCAX and sporadic cases to enhance the cytotoxicity of DNA-damaging agents or radiotherapy. Research groups are working to identify the small molecule that is able to target the DNA repair activity of APEX1/REF1, which could enter into clinical trials. Given that the role of XRCC1 in the NHEJ pathway and our results showed no change in PARP1 expression and downregulation of XRCC1 in BRCA2-related patients, targeting NHEJ could be considered an alternative synthetic lethal approach. 
Moreover, in BRCAX patients, XRCC1 could be evaluated as a molecular target. The overexpression of PARP1 and miR17 in mesenchymal-like cell lines and triple-positive sporadic cancers would suggest validating them as molecular biomarkers to identify patients with the BRCAness phenotype. Preclinical studies on sensitivity to PARP inhibitors and radiotherapy are underway to confirm what the expression data has suggested.

\section{Materials and Methods}

Cell lines and culture. BRCA1-proficient, triple-negative human breast carcinoma MDA-MB231 cells were grown in DMEM supplemented with $10 \%$ fetal bovine serum (FBS) and $2 \mathrm{~mm}$ L-glutamine at $37^{\circ} \mathrm{C}$ in $5 \% \mathrm{CO}_{2}$. SUM149PT cells were isolated from a patient with triple-negative inflammatory breast cancer who carried the truncating mutation 2288delT in BRCA1 and whose disease progressed after chemotherapy treatment. SUM149PT were grown in F-12 HAM's nutrient mixture (EuroClone SpA, Pero, Milano, Italy) supplemented with $5 \% \mathrm{FBS}, 5 \mu \mathrm{g} / \mathrm{ml}$ insulin (Sigma-Aldrich, St. Louis, MO, USA), and $1 \mu \mathrm{g} / \mathrm{ml}$ hydrocortisone (SigmaAldrich) at $37^{\circ} \mathrm{C}$ in $5 \% \mathrm{CO}_{2}$. SUM1315MO2 is an ER-positive cell line, isolated from a highly invasive breast tumor that was transplanted in immune-deficient mice for two generations. These cells carry the BRCA1 185delAG pathogenetic mutation. SUM1315MO2 were grown in F-12 HAM's nutrient mixture (EuroClone SpA) supplemented with $5 \% \mathrm{FBS}, 5 \mu \mathrm{g} / \mathrm{ml}$ insulin (Sigma-Aldrich), and $10 \mathrm{ng} / \mathrm{ml}$ epidermal growth factor (Sigma-Aldrich) at $37^{\circ} \mathrm{C}$ in $5 \% \mathrm{CO}_{2}$.

Patients. Forty-three patients were enrolled -27 with familial breast cancer (10 BRCA1-mutated, 4 BRCA2-mutated, and 13 wild type for both genes and defined as BRCAX patients) and 16 with sporadic breast cancer - through the Genetic Counseling Program at NCRC 'Giovanni Paolo II' in Bari, Italy. Familial patients were considered as those with clinical criteria of familiarity, as defined previously, ${ }^{42}$ and those with a high risk of carrying the BRCA1/2 mutation as evaluated by the BRCAPRO software (BayesMendel Software, Dana-Farber Cancer Institute, Boston, MA, USA). All patients signed informed consent.

All familial patients were analyzed for the mutational status of BRCA1/2 genes as reported previously. ${ }^{20}$ Tumor staging was performed according to the UICC TNM

Table 2 Clinicopathological features of familial and sporadic breast cancer (BC) cohorts

\begin{tabular}{|c|c|c|c|c|}
\hline & \multicolumn{3}{|c|}{ Familial BCs } & \multirow{2}{*}{$\begin{array}{c}\text { Sporadic BCs, } \\
\quad n=16\end{array}$} \\
\hline & $\begin{array}{c}\text { Mutated } \\
\text { BCs, } \\
n=14\end{array}$ & $\begin{array}{c}\text { BRCAX } \\
\text { BCs, } \\
n=13\end{array}$ & $\begin{array}{c}\text { Tot, } \\
n=27\end{array}$ & \\
\hline $\begin{array}{l}\text { Median age (years) } \\
\text { (range) }\end{array}$ & $\begin{array}{c}45 \\
(35-59)\end{array}$ & $\begin{array}{c}48 \\
(33-68)\end{array}$ & $\begin{array}{c}46 \\
(33-48)\end{array}$ & $\begin{array}{c}56 \\
(40-76)\end{array}$ \\
\hline $\begin{array}{l}\text { Histopathology } \\
\text { IDC } \\
\text { ILC } \\
\text { In situ }\end{array}$ & 14 & 13 & 27 & $\begin{array}{r}13 \\
2 \\
1\end{array}$ \\
\hline $\begin{array}{l}T \\
\text { T1-2 } \\
\text { T3-4 }\end{array}$ & 14 & $\begin{array}{r}12 \\
1\end{array}$ & $\begin{array}{r}26 \\
1\end{array}$ & $\begin{array}{r}11 \\
5\end{array}$ \\
\hline $\begin{array}{l}N_{\mathrm{NO}} \\
\mathrm{N}+\end{array}$ & $\begin{array}{l}6 \\
8\end{array}$ & $\begin{array}{l}6 \\
7\end{array}$ & $\begin{array}{l}12 \\
15\end{array}$ & $\begin{array}{r}4 \\
12\end{array}$ \\
\hline $\begin{array}{l}\text { G } \\
\text { G1 } \\
\text { G2 } \\
\text { G3 }\end{array}$ & $\begin{array}{l}5 \\
9\end{array}$ & $\begin{array}{l}5 \\
8\end{array}$ & $\begin{array}{l}10 \\
17\end{array}$ & $\begin{array}{l}3 \\
6 \\
7\end{array}$ \\
\hline $\begin{array}{l}\text { ER }+ \\
\text { PgR }+ \\
\text { HER2 }+ \\
\text { Mib-1>10 }\end{array}$ & $\begin{array}{l}7 \\
7 \\
5 \\
6\end{array}$ & $\begin{array}{r}5 \\
6 \\
3 \\
11\end{array}$ & $\begin{array}{r}12 \\
13 \\
8 \\
17\end{array}$ & $\begin{array}{r}15 \\
10 \\
2 \\
12\end{array}$ \\
\hline
\end{tabular}

classification. The clinicopathological characteristics of the patients are reported in Table 2. Tissue samples, collected during surgical resection, were stored at $-80^{\circ} \mathrm{C}$ in the Institutional Tissue Bank of NCRC 'Giovanni Paolo II' in Bari, Italy.

RNA extraction and cDNA synthesis. RNA was extracted from cell lines and both malignant and healthy fresh-frozen tissue samples with the RNeasy Plus Mini Kit (Qiagen, Hilden, Germany) as indicated by the manufacturer and quantified through NanoDrop8000 (Thermo Fisher Scientific Inc., Waltham, MA, USA). RNA extracted from healthy tissues were mixed in an equimolar manner to obtain pools of RNA from BRCA1-mutated, BRCA2-mutated, BRCAX, and sporadic cases, which was useful to normalize data. Five hundred nanograms of RNA were retrotranscribed through the High-Capacity cDNA Reverse Transcription Kit (Applied Biosystems, Foster City, CA, USA) as indicated by the manufacturer.

Real-time PCR. Analysis of expression was performed using a fluorescencebased, real-time detection method (TaqMan probes; Applied Biosystems) on the ABI PRISM 7000 Sequence Detection System (Applied Biosystems). Probes were directed to REF1/APEX1 (Hs00172396 m1), XRCC1 (Hs00959834 m1), NTHL1 (Hs00959764_m1), OGG1 (Hs00213454_m1), and PARP1 (Hs00242302_m1). The selected assays were designed across an exon-exon junction to produce a short amplicon that gives a more efficient PCR reaction. A $20 \mu \mathrm{l}$ PCR reaction including $2.5 \mu \mathrm{l}$ of RT product, $1 \times$ TaqMan Universal PCR Master Mix, and $1 \times$ of the corresponding TaqMan Gene Expression Assay was incubated in 96-well plates at $95^{\circ} \mathrm{C}$ for $10 \mathrm{~min}$, followed by 40 cycles of $95^{\circ} \mathrm{C}$ for $15 \mathrm{~s}$ and $60^{\circ} \mathrm{C}$ for $1 \mathrm{~min}$. PCR reactions were performed in triplicate including no-template controls. Relative quantities of each cDNA were calculated using the $\Delta \Delta \mathrm{Ct}$ method after normalization with endogenous reference 18s rRNA (Hs03928985_g1) and calibrating $\mathrm{Ct}$ values with respect to the $\mathrm{Ct}$ of healthy tissues.

miRNA study. The levels of hsa-miR-17 were measured using the TaqMan microRNA Assay (Applied Biosystems, Foster City, CA, USA) on the Applied Biosystems real-time PCR instrument 7000 in accordance with the manufacturer's instructions. RNU 48 was used to normalize miRNA levels. A $20 \mu \mathrm{l} \mathrm{PCR} \mathrm{reaction}$ including $3 \mu \mathrm{l}$ of RT product, $1 \times$ TaqMan Universal PCR Master Mix, and $1 \times$ of the corresponding miRNA assay primers was incubated in 96 -well plates at $95^{\circ} \mathrm{C}$ for $10 \mathrm{~min}$, followed by 40 cycles of $95^{\circ} \mathrm{C}$ for $15 \mathrm{~s}$ and $60^{\circ} \mathrm{C}$ for $1 \mathrm{~min}$. PCR reactions were performed in triplicate including no-template controls. Relative quantities of each miRNA were calculated using the $\Delta \Delta \mathrm{Ct}$ method after normalization with endogenous reference RNU 48.

Dual-luciferase reporter assay and constructs. The luciferase-UTR reporter plasmid was constructed by introducing the APEX1 3'-UTR (NM_001641) into pmiR-REPORT miRNA Expression Reporter Vector System (Life Technologies, Foster City, CA, USA). The APEX $13^{\prime}$-UTR sequence was amplified by PCR from HEK293-T cDNA. Mutagenesis was used to delete miR-17 binding site using the QuickChange II Kit (Stratagene, La Jolla, CA, USA). All constructs were verified by sequencing. The reporter constructs, pSV-Renilla (pRL-SV40; Promega, Fitchburg, WI, USA) and miR-17 mimic (Dharmacon, Lafayette, CO, USA; Sigma-Aldrich), were transfected into HEK293 cells using Lipofectamine 2000 (Life Technologies). After $48 \mathrm{~h}$, the cells were lysed in passive lysis buffer and assayed for both firefly and Renilla luciferase activity using the Dual-GLO Luciferase Assay System (Promega). Firefly luciferase activity was normalized to Renilla luciferase activity for each transfected well. Values are the mean \pm s.e.m. of three experimental replicates from two to four independent transfections. Significance was determined by a two-tailed unpaired $t$-test for means.

Computational and statistical analysis. Predicted targets of miR17 were analyzed through the miRWalk database (http: //www.ma.uni-heidelberg.de/ appsz/zmf/mirwalk/). miRWalk consists of two modules: the predicted target module, which presents results from eight established miRNA prediction programs (i.e., RNA22, miRanda, miRDB, TargetScan, PITA, and Diana-microT), and the validated targets module, which hosts new and unique features on experimentally validated miRNA interaction information. Relative amounts of transcripts were calculated with the $\Delta \Delta \mathrm{Ct}$ method after normalization with endogenous control. Median values were used as cutoff for all enzyme transcripts and for hsa-miR-17 to discriminate between low- and overexpressing patients. The Mann-Whitney $U$-test was performed to compare relative expression. The statistical association between mutation frequency and the other parameters was assessed using a $\chi^{2}$ 
test and the two-tailed Fisher's test. Because of the small dimension of BRCA1and BRCA2-related breast cancer samples, we considered them together in the statistical analysis.

Differences were considered to be significant when the $P$-value was $<0.05$. Statistical analyses were carried out with the SPSS statistical software v. 17 (SPSS Inc., Chicago, IL, USA).

\section{Conflict of Interest}

The authors declare no conflict of interest.

Acknowledgements. We thank Caroline Oakley for manuscript revision. This work was partially funded by Regione Puglia (DIEF 2011) and by RF 2008 Ministry of Health.

1. Bielas JH, Loeb KR, Rubin BP, True LD, Loeb LA. Human cancers express a mutator phenotype. Proc Natl Acad Sci USA 2006; 103: 18238-18242.

2. Lengauer $\mathrm{C}$, Kinzler KW, Vogelstein B. Genetic instabilities in human cancers. Nature 1998; 396: 643-649.

3. Vogelstein B, Kinzler KW. Cancer genes and the pathways they control. Nat Med 2004; 10: 789-799.

4. Chacon RD, Costanzo MV. Triple-negative breast cancer. Breast Cancer Res 2010; 12(Suppl 2): S3.

5. Walsh T, Casadei S, Coats KH, Swisher E, Stray SM, Higgins J et al. Spectrum of mutations in BRCA1, BRCA2, CHEK2, and TP53 in families at high risk of breast cancer. JAMA 2006; 295: 1379-1388.

6. Prat A, Perou CM. Deconstructing the molecular portraits of breast cancer. Mol Oncol 2011; 5: 5-23.

7. Kerr P, Ashworth A. New complexities for BRCA1 and BRCA2. Curr Biol 2001; 11: R668-R676.

8. Venkitaraman AR. Cancer susceptibility and the functions of BRCA1 and BRCA2. Cell 2002; 108: 171-182.

9. Wang Y, Cortez D, Yazdi P, Neff N, Elledge SJ, J Qin. BASC, a super complex of BRCA1associated proteins involved in the recognition and repair of aberrant DNA structures. Genes Dev 2000; 14: 927-939.

10. Le Page F, Randrianarison V, Marot D, Cabannes J, Perricaudet M, Feunteun $\mathrm{J}$ et al. BRCA1 and BRCA2 are necessary for the transcription-coupled repair of the oxidative 8-oxoguanine lesion in human cells. Cancer Res 2000; 60: 5548-5552.

11. Zhong $Q$, Boyer TG, Chen PL, Lee WH. Deficient nonhomologous end-joining activity in cell-free extracts from Brca1-null fibroblasts. Cancer Res 2002; 62: 3966-3970.

12. Hartman AR, Ford JM. BRCA1 induces DNA damage recognition factors and enhances nucleotide excision repair. Nat Genet 2002; 32: 180-184.

13. Saha T, Rih JK, Roy R, Ballal R, Rosen EM. Transcriptional regulation of the base excision repair pathway by BRCA1. J Biol Chem 2010; 285: 19092-19105.

14. Abbott DW, Freeman ML, Holt JT. Double-strand break repair deficiency and radiation sensitivity in BRCA2 mutant cancer cells. J Natl Cancer Inst 1998; 90: 978-985.

15. Bogliolo M, Taylor RM, Caldecott KW, Frosina G. Reduced ligation during DNA base excision repair supported by BRCA2 mutant cells. Oncogene 2000; 19: 5781-5787.

16. Xu XR, Gammon MD, Zhang YJ, Bestor TH, Zeisel SH, Wetmur JG et al. BRCA1 promoter methylation is associated with increased mortality among women with breast cancer Breast Cancer Res Treat 2009; 115: 397-404.

17. Kutanzi KR, Yurchenko OV, Beland FA, Checkhun VF, Pogribny IP. MicroRNA-mediated drug resistance in breast cancer. Clin Epigenet 2011; 2: 171-185.

18. Lehmann BD, Bauer JA, Chen $X$, Sanders ME, Chakravarthy AB, Shyr $Y$ et al. Identification of human triple-negative breast cancer subtypes and preclinical models for selection of targeted therapies. J Clin Invest 2011; 121: 2750-2767.

19. Grigoriadis A, Mackay A, Noel E, Wu PJ, Natrajan R, Frankum J et al. Molecular characterisation of cell line models for triple-negative breast cancers. BMC Genom 2012; 13: 619 .

20. Bobola MS, Blank A, Berger MS, Stevens BA, Silber JR. Apurinic/apyrimidinic endonuclease activity is elevated in human adult gliomas. Clin Cancer Res 2001; 7: 3510-3518.

21. Thomson B, Tritt R, Davis M, Kelley MR. Histology-specific expression of a DNA repair protein in pediatric rhabdomyosarcomas. J Pediatr Hematol/Oncol 2001; 23: 234-239.

22. Puglisi F, Aprile G, Minisini AM, Barbone F, Cataldi P, Tell G et al. Prognostic significance of Ape1/ref-1 subcellular localization in non-small cell lung carcinomas. Anticancer Res 2001; 21: 4041-4049.

23. Koukourakis MI, Giatromanolaki A, Kakolyris S, Sivridis E, Georgoulias V, Funtzilas G et al. Nuclear expression of human apurinic/apyrimidinic endonuclease (HAP1/REF-1) in head-and-neck cancer is associated with resistance to chemoradiotherapy and poor outcome. Int J Radiat Oncol 2001; 50: 27-36.

24. Robertson KA, Bullock HA, Xu Y, Tritt R, Zimmerman E, Ulbright TM et al. Altered expression of Ape1/ref-1 in germ cell tumors and overexpression in NT2 cells confers resistance to bleomycin and radiation. Cancer Res 2001; 61: 2220-2225.

25. Wu HH, Chu YC, Wang L, Tsai LH, Lee MC, Chen CY et al. Cytoplasmic Ape1 expression elevated by p53 aberration may predict survival and relapse in resected non-small cell lung cancer. Ann Surg Oncol 2012; 20(Suppl 3): S336-S347.

26. Barzilay G, Walker LJ, Robson CN, Hickson ID. Site-directed mutagenesis of the human DNA repair enzyme HAP1: identification of residues important for AP endonuclease and RNase H activity. Nucleic Acids Res 1995; 23: 1544-1550.

27. Luo M, Kelley MR. Inhibition of the human apurinic/apyrimidinic endonuclease (APE1) repair activity and sensitization of breast cancer cells to DNA alkylating agents with lucanthone. Anticancer Res 2004; 24: 2127-2134.

28. Fishel ML, He Y, Smith ML, Kelley MR. Manipulation of base excision repair to sensitize ovarian cancer cells to alkylating agent temozolomide. Clin Cancer Res 2007; 13: 260-267.

29. Liu L, Nakatsuru Y, Gerson SL. Base excision repair as a therapeutic target in colon cancer. Clin Cancer Res 2002; 8: 2985-2991.

30. Taverna P, Liu LL, Hwang HS, Hanson AJ, Kinsella TJ, Gerson SL. Methoxyamine potentiates DNA single strand breaks and double strand breaks induced by temozolomide in colon cancer cells. Mutat Res-DNA Repair 2001; 485: 269-281.

31. Alli E, Sharma VB, Sunderesakumar P, Ford JM. Defective repair of oxidative dna damage in triple-negative breast cancer confers sensitivity to inhibition of poly(ADP-ribose) polymerase. Cancer Res 2009; 69: 3589-3596.

32. Fong PC, Boss DS, Yap TA, Tutt A, Wu PJ, Mergui-Roelvink M et al. Inhibition of poly (ADP-Ribose) polymerase in tumors from BRCA mutation carriers. N Engl J Med 2009; 361: 123-134.

33. Chuang HC, Kapuriya N, Kulp SK, Chen CS, Shapiro CL. Differential anti-proliferative activities of poly(ADP-ribose) polymerase (PARP) inhibitors in triple-negative breast cancer cells. Breast Cancer Res Treat 2012; 134: 649-659.

34. Goncalves A, Finetti P, Sabatier R, Gilabert M, Adelaide J, Borg JP et al. Poly(ADP-ribose) polymerase-1 mRNA expression in human breast cancer: a meta-analysis. Breast Cancer Res Treat 2011; 127: 273-281.

35. Turner NC, Reis JS, Russell AM, Springall RJ, Ryder K, Steele D et al. BRCA1 dysfunction in sporadic basal-like breast cancer. Oncogene 2007; 26: 2126-2132.

36. Ladiges WC. Mouse models of XRCC1 DNA repair polymorphisms and cancer. Oncogene 2006; 25: 1612-1619.

37. Nazarkina ZK, Khodyreva SN, Marsin S, Radicella JP, Lavrik OI. Study of interaction of XRCC1 with DNA and proteins of base excision repair by photoaffinity labeling technique. Biochemistry (Moscow) 2007; 72: 878-886.

38. Weaver DA, Crawford EL, Warner KA, Elkhairi F, Khuder SA, Willey JC. ABCC5, ERCC2, $\mathrm{XPA}$ and XRCC1 transcript abundance levels correlate with cisplatin chemoresistance in non-small cell lung cancer cell lines. Mol Cancer 2005; 4: 18.

39. Sultana R, Abdel-Fatah T, Abbotts R, Hawkes C, Albarakati N, Seedhouse C et al. Targeting XRCC1 deficiency in breast cancer for personalized therapy. Cancer Res 2013; 73: $1621-1634$.

40. Wang HC, Perrault AR, Takeda Y, Qin W, Wang HY, lliakis G. Biochemical evidence for Ku-independent backup pathways of NHEJ. Nucleic Acids Res 2003; 31: 5377-5388.

41. Dibiase SJ, Zeng ZC, Chen R, Hyslop T, Curran WJ, lliakis G. DNA-dependent protein kinase stimulates an independently active, nonhomologous, end-joining apparatus. Cancer Res 2000; 60: 1245-1253.

42. Tommasi S, Pilato B, Pinto R, Monaco A, Bruno M, Campana M et al. Molecular and in silico analysis of BRCA1 and BRCA2 variants. Mutat Res 2008; 644: 64-70.

43. Peasland A, Wang LZ, Rowling E, Kyle S, Chen T, Hopkins A et al. Identification and evaluation of a potent novel ATR inhibitor, NU6027, in breast and ovarian cancer cell lines. Br J Cancer 2011; 105: 372-381.

(i) (-) () Cell Death and Disease is an open-access journal published by Nature Publishing Group. This work is licensed under a Creative Commons Attribution-NonCommercialNoDerivs 4.0 International License. The images or other third party material in this article are included in the article's Creative Commons license, unless indicated otherwise in the credit line; if the material is not included under the Creative Commons license, users will need to obtain permission from the license holder to reproduce the material. To view a copy of this license, visit http://creativecommons.org/licenses/ by-nc-nd/4.0/ 